TITLE:

\title{
Anomalous ionization efficiency by few-cycle pulses in the multiphoton ionization regime
}

$\operatorname{AUTHOR}(S):$

Liu, C; Nakajima, T

CITATION:

Liu, C ...[et al]. Anomalous ionization efficiency by few-cycle pulses in the multiphoton ionization regime. PHYSICAL REVIEW A 2007, 76(2): 023416.

ISSUE DATE:

2007-08

URL:

http://hdl.handle.net/2433/50424

RIGHT:

Copyright 2007 American Physical Society 
PHYSICAL REVIEW A 76, 023416 (2007)

\title{
Anomalous ionization efficiency by few-cycle pulses in the multiphoton ionization regime
}

\author{
Chengpu Liu and Takashi Nakajima* \\ Institute of Advanced Energy, Kyoto University, Gokasho, Uji, Kyoto 611-0011, Japan
}

(Received 22 May 2007; published 20 August 2007)

\begin{abstract}
We theoretically study the effect of laser pulse durations on the ionization yield of hydrogen atoms and negative hydrogen ions. By numerically solving the time-dependent Schrödinger equation we find that the ionization efficiency by few-cycle pulses is unexpectedly larger than that by many-cycle pulses at low intensities when multiphoton ionization is the dominant ionization mechanism.
\end{abstract}

DOI: 10.1103/PhysRevA.76.023416

PACS number(s): $32.80 . \mathrm{Rm}, 32.80 . \mathrm{Fb}, 32.80 . \mathrm{Gc}, 42.50 . \mathrm{Hz}$

When the laser intensity is sufficiently high, nonlinear phenomena are induced upon interaction with a matter. Multiphoton ionization is one of such processes [1]. To understand the ionization dynamics, there are two essential parameters we need to take into account in addition to the laser wavelength: one is the laser intensity and the other is the laser pulse duration. At lower intensities with rather long (from tens of ns to ps) pulse duration, typically below $\sim 10^{11} \mathrm{~W} / \mathrm{cm}^{2}$, the lowest order time-independent perturbation theory (LOPT) gives a good prediction in terms of multiphoton ionization cross sections, if (near-) resonant transitions do not take place. If, on the other hand, there occur (near-) resonant transitions, one must perform a timedependent analysis in terms of amplitude equations or density matrix equations with a few essential states. As the laser intensity further increases the nonperturbative response set in. When the intensity is even higher, typically $>10^{13} \mathrm{~W} / \mathrm{cm}^{2}$, the pulse duration must be necessarily shorter than the few ps for the visible laser wavelengths to avoid complete ionization, since, otherwise, atoms cannot see the peak intensity. For such high intensities it is well known that tunneling ionization or barrier suppression ionization takes place, and the system behavior becomes strongly nonperturbative [2]. Mathematically speaking, this means that the time-dependent wave function cannot be represented in terms of only a few essential states. A complete wave function would be necessary to represent the time evolution of the system.

Recent technological advances in ultrafast optics have permitted the generation of light pulse comprising only a few cycles of the electric field [3-5], where the few-cycle effects originating from the carrier-envelope phase (CEP) of the pulse plays an important role in the physical processes such as above-threshold ionization [6] and high harmonic generation [3,7], etc. Although it is widely believed that the CEP effects can be seen only in the strong field (tunneling ionization, TI) regime, we have recently shown that they can be seen even in the weak field (multiphoton ionization, MI) regime where the physical origin of the phase dependence is completely different $[8,9]$. That is, there is an essential

*t-nakajima@iae.kyoto-u.ac.jp difference in the dynamics induced by few-cycle pulses under the two different intensity regimes, i.e., MI and TI regime: For few-cycle pulses in the MI regime, the description of the system requires a complete wave function due to the extremely broad spectral bandwidth and thus infinitely many states participate the interaction, while for few-cycle pulses in the TI regime the system behavior becomes nonperturbative and a complete wave function is again needed due to the field strength comparable to the atomic potential.

Having understood the above, it is clear now that the manifestation of the few-cycle effects in the MI regime is not only limited to the CEP effects, since few-cycle effects in the MI regime essentially come from the extremely broad spectral bandwidth of the pulse. In contrast, few-cycle effects in the TI regime come from the field strength itself.

The purpose of this paper is to theoretically show that, even if the CEP of the few-cycle pulse is not stabilized, we find an anomaly in the ionization efficiency for few-cycle pulses when the intensity is within the MI regime. Our theoretical tool is a time-dependent Schrödinger equation (TDSE). By comparing the numerical results for the hydrogen atom and the negative hydrogen ion, we can also clarify the influence of the bound states on the ionization efficiency by few-cycle pulses.

The TDSE we now solve is in the form of

$$
i \frac{\partial \Psi(t)}{\partial t}=\left[H_{0}+V(t)\right] \Psi(t)
$$

where $H_{0}$ is the field-free atomic Hamiltonian and $V(t)$ is the time-dependent interaction between the electron and the laser field, which is defined by $V(t)=\hat{\varepsilon} \cdot r E(t)$, with $E(t)$ being the electric field, $\hat{\varepsilon}$ the polarization vector, and $r$ the position operator of the valence electron. The atomic units (a.u., $m$ $=\hbar=e=1)$ are used in all equations in this paper, unless otherwise mentioned. For the following numerical calculations, we define the vector potential as 


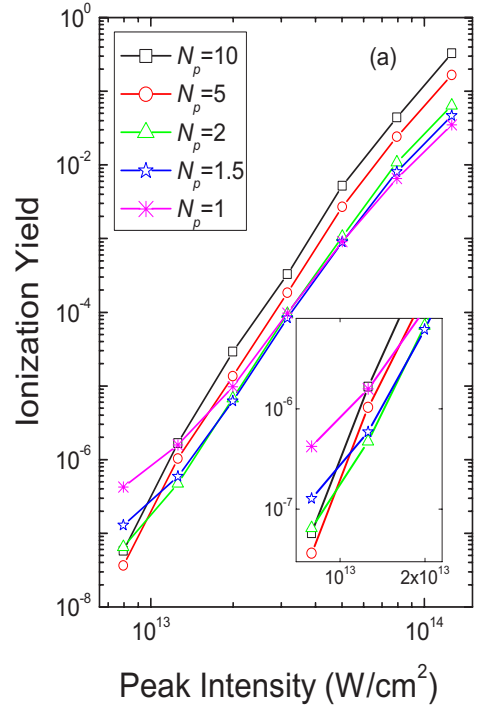

$$
A(t)=A_{0} f(t) \cos (\omega t+\Phi)= \begin{cases}A_{0} \sin ^{2}\left(\frac{\pi t}{2 \tau_{p}}\right) \cos (\omega t+\Phi) & \text { (sine-squared pulse) }, \\ A_{0} \exp \left[-4 \ln 2\left(\frac{t}{\tau_{p}}\right)^{2}\right] \cos (\omega t+\Phi) & \text { (Gaussian pulse) } .\end{cases}
$$

Here $A_{0}$ is the peak amplitude of the vector potential, $f(t)$ the field envelope, $\omega$ the photon energy, and $\Phi$ the CEP. $\tau_{p}$ is a duration [full width at half maximum (FWHM)] of the pulse with the definition of $\tau_{p}=2 \pi N_{p} / \omega$, where $N_{p}$ is a number of cycles of the pulse and can be a noninteger number. It is worth noting that, for very short laser pulses including only a few cycles, say one or two, the field envelope may change significantly within a single optical period. In such a case, one should first define the vector potential, $A(t)$, and then use $E(t)=-\partial A(t) / \partial t$ to obtain the electric field, $E(t)$. This guarantees that $A(t)$ vanishes at $t= \pm \infty$, or equivalently the pulse area under the $E(t)$ function becomes exactly zero after integrating $E(t)$ over the entire pulse duration [10]. Otherwise, unphysical results may be obtained. From the vector potential, we obtain

$$
E(t)=A_{0} \omega \sin (\omega t+\Phi)-A_{0} \frac{\partial f(t)}{\partial t} \cos (\omega t+\Phi)
$$

Note that the second term which consists of the time derivative of the vector potential envelope is negligible for manycycle pulses, while for few-cycle pulses, this term plays an import role in the physical processes.

First we show the results for the $\mathrm{H}$ atom. The screened soft-core potential model, $V(x)=-\alpha / \sqrt{1+x^{2}}$, is employed for the one-dimensional (1D) numerical calculation of TDSE [11]. For the $1 \mathrm{D} \mathrm{H}$ atom, $\alpha$ is set to be 0.775 [12], which supports eigenenergy of $-13.6 \mathrm{eV}$ for the ground state. Figure 1(a) shows the ionization yield as a function of peak intensity at the wavelength of $800 \mathrm{~nm}$ for different numbers of cycles, $N_{p}=10,5,2,1.5$, and 1 , with a sine-squared temporal envelope. Note that the results for $N_{p}=1,1.5$, and 2 have been averaged over different CEP's, which somehow mimics the few-cycle pulse without phase-stabilization. For longer pulses, we have chosen the CEP equal to 0 , since we know that there is practically no phase dependence. For $N_{p}$ $=10$, the curve (black line with square) appears as an almost straight line, up to the peak intensity of $5.0 \times 10^{13} \mathrm{~W} / \mathrm{cm}^{2}$, with a slope of $\sim 5.5$, indicating that our result agrees well with the prediction of LOPT. For the further increment of peak intensity, the slope becomes smaller, indicating that the saturation starts to take place. For $N_{p}=5$ (red line with circle), the ionization behavior is similar to that for $N_{p}=10$. The two curves are practically parallel, which implies that ionization by the longer pulse is more efficient. If shorter pulses are used, however, we find an anomalous behavior: For example, see the results for $N_{p}=2,1.5$, and 1 in Fig. 1(a). In the lower intensity region [inset of Fig. 1(a)], the ionization yield for shorter pulses turns out to be larger than that for longer pulses. This implies that shorter pulses are more efficient to induce ionization than longer pulses, provided that the peak intensity is chosen to be the same for all pulse durations. These results seem to imply that the spectral bandwidth plays a more important role in the lower intensity region. With the increment of intensity, such an ionization anomaly gradually disappears.

Since the sine-squared pulse is known to have a much broader spectral wing compared with, say, a Gaussian pulse with the same pulse duration, we now need to examine the effect of the temporal pulse shape for few-cycle pulses in terms of ionization efficiency. Related to this, for the case of 


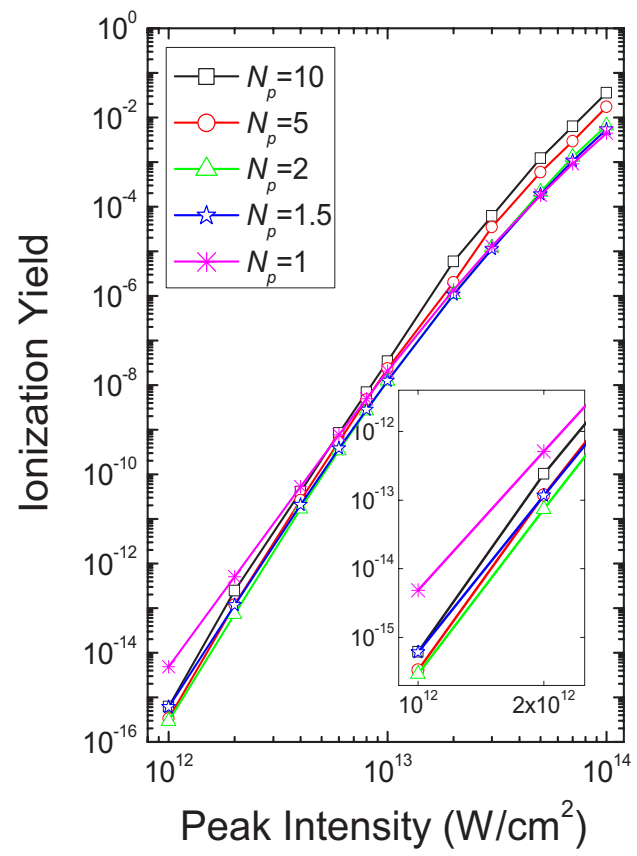

FIG. 2. (Color online) Ionization yield of the three-dimensional $\mathrm{H}$ atom as a function of peak intensity for different numbers of cycles. The pulse shape is Gaussian and the laser wavelength is $800 \mathrm{~nm}$.

many-cycle pulses we have recently examined the pulse shape effects on the above-threshold ionization spectra of $\mathrm{Mg}$ [13]. As a matter of fact, a Gaussian pulse is more realistic than a sine-squared pulse. The results for the Gaussian pulses are shown in Fig. 1(b). As we expect, the ionization efficiency is a little bit smaller for the Gaussian pulses than the sine-squared pulses, in particular in the lower intensity region, due to the reason explained above. Note, however, that we can still see the anomaly.

Observation of the ionization anomaly in the lower intensity region is not an artifact of the use of the $1 \mathrm{D} \mathrm{H}$ atom. To verify this, we have also performed the three-dimensional (3D) calculation for the $\mathrm{H}$ atom $[9,14]$. The results are shown in Fig. 2 for the Gaussian pulses. It is clear that the tendency is very similar to that of the $1 \mathrm{D}$ calculations and the ionization anomaly is again clearly seen. There is, however, a remark to be made for the $1 \mathrm{D}$ and 3D calculations. Comparing the results of $1 \mathrm{D}$ and $3 \mathrm{D}$ for the Gaussian pulses with long pulse durations, say, $N_{p}=10$ in Fig. 1(b) and Fig. 2, the slope by the $1 \mathrm{D}$ calculation is 5.5 , while that for the $3 \mathrm{D}$ calculation is 8.8. This can be mainly attributed to the difference of the level structure: the location of the first excited state of the 1D $\mathrm{H}$ atom is $-5.03 \mathrm{eV}$, which is about $1.5 \mathrm{eV}$ closer to the ground state than that of the $3 \mathrm{D} \mathrm{H}$ atom. Therefore, depending on the physical quantities to look at, the limitation of the $1 \mathrm{D}$ calculation must be kept in mind if one is to quantitatively compare 1D results with experimental data.

As we have mentioned above, the fact that the ionization anomaly is seen only in the MI regime seems to imply that its physical origin would be a very broad spectral bandwidth of the few-cycle pulse, since the spectral bandwidth plays a lesser role in the TI regime. Besides, the ponderomotive shifts become significant at the higher intensity. This consideration has led us to the speculation that, if the anomaly comes from the spectral bandwidth of the pulse, the appearance of the ionization anomaly is not accidental and should be seen in any atoms or ions, including those without excited bound states.

To verify this, we now study the negative hydrogen ion, $\mathrm{H}^{-}$, since the ground state is the only bound state. In the theoretical studies, a zero-range [15] or short-range $[16,17]$ potential model is commonly used. In the following, we have employed the short-range potential model: $V(x)$ $=-\exp (-|x|) / \sqrt{\alpha^{2}+x^{2}}$, where $\alpha$ is chosen to be 6.27. Figures $3(\mathrm{a})$ and 3 (b) show the detachment yield of $1 \mathrm{D} \mathrm{H}^{-}$ion as a function of peak intensity at the wavelength of $4 \mu \mathrm{m}$ for different numbers of cycles with the sine-squared and Gaussian pulses, respectively. The tendency turns out to be very similar to those shown in Figs. 1(a) and 1(b). For further confirmation we have performed calculations for the $3 \mathrm{D} \mathrm{H}^{-}$ ion and present the results in Fig. 4. For simplicity we have employed the Yukawa potential with a single-active electron approximation [18]: $V(r)=-1.1 \exp (-r) / r$, which yields the correct binding energy of $\mathrm{H}^{-}$ion, $-0.754 \mathrm{eV}$. Clearly the

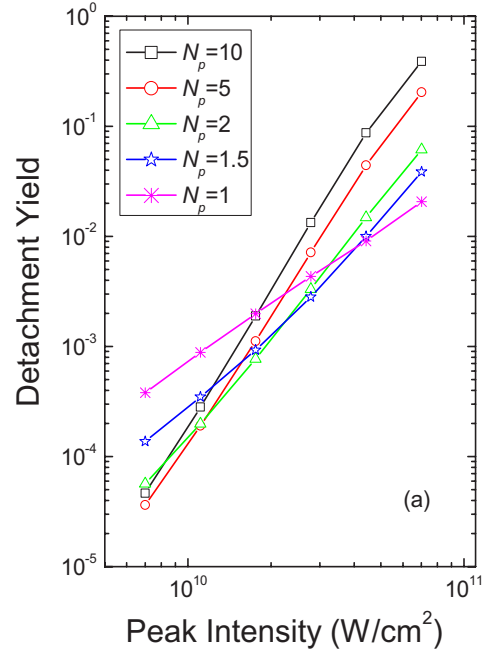

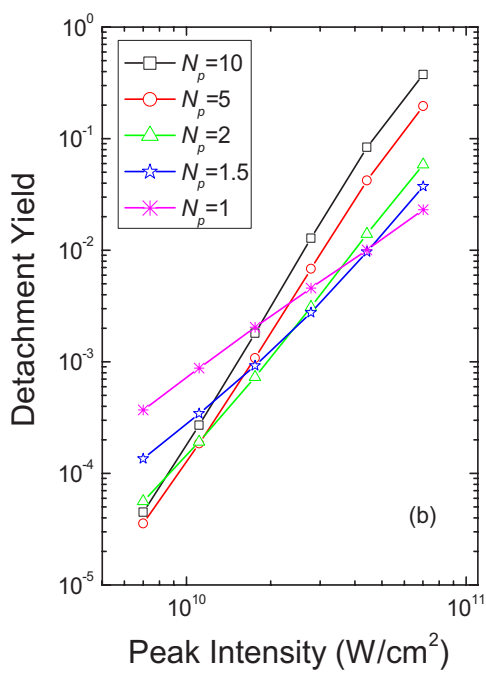

FIG. 3. (Color online) Detachment yield of the one-dimensional $\mathrm{H}^{-}$ion as a function of peak intensity for different numbers of cycles with the (a) sine-squared and (b) Gaussian pulses. The laser wavelength is $4 \mu \mathrm{m}$. 


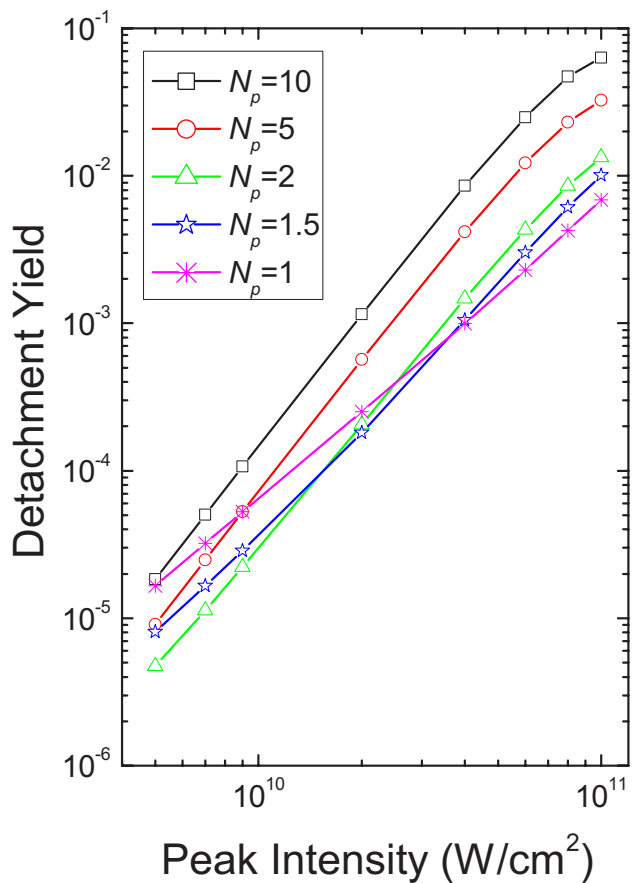

FIG. 4. (Color online) Detachment yield of the threedimensional $\mathrm{H}^{-}$ion as a function of laser intensity for different numbers of cycles. The pulse shape is Gaussian and the laser wavelength is $4 \mu \mathrm{m}$.

similar tendency persists. Based on those findings for the 1D and $3 \mathrm{D} \mathrm{H}^{-}$ions, it is now clear that the ionization anomaly for $N_{p}=1-2$ cases is not due to the presence of intermediate bound states.

From the above calculations, we have confirmed that the ionization anomaly is rather general: no matter what atoms and/or ions we employ, the ionization anomaly will be seen, as long as the laser pulse is sufficiently short. After a number of computer runs (not shown here), we have confirmed that the anomaly would be more striking if more numbers of photons are involved to ionize. It is clear now that, when the pulse duration is extremely short, $n$-photon ionization becomes $n^{\prime}(<n)$-photon ionization due to the extremely broad spectral bandwidth of the pulse, and as a result, the ionization efficiency by fewer-cycle pulses becomes larger than that by longer-cycle pulses in the MI regime. As the intensity increases, the spectral bandwidth of the pulse plays a lesser and lesser role due to the more and more contribution of tunneling ionization, and the ionization anomaly gradually disappears.

In conclusion, we have theoretically investigated the ionization anomaly of the hydrogen atom and negative hydrogen ion by few-cycle pulses as a function of peak intensity for different pulse durations and different temporal pulse shapes. We have found that the ionization efficiency by fewer-cycle pulses can be larger than that by more than a few-cycle pulses in the low intensity region where multiphoton ionization is the dominant ionization mechanism. This is due to the extremely broad spectral bandwidths of fewercycle pulses, and should be found for any atoms, ions, or molecules. In the higher intensity region where tunneling ionization takes place, the spectral bandwidths of the fewcycle pulses play a lesser role for ionization, and as a result the anomaly disappears.

C.L. acknowledges financial support from the Japan Society for the Promotion of Sciences (JSPS) and the hospitality at Kyoto University during his stay. The work by T.N. was supported by a Grant-in-Aid for scientific research from the Ministry of Education and Science of Japan.
[1] F. H. M. Faisal, Theory of Multiphoton Processes (Plenum Press, New York, 1987).

[2] P. Lambropoulos, P. Maragakis, and J. Zhang, Phys. Rep. 305, 203 (1998).

[3] T. Brabec and F. Krauze, Rev. Mod. Phys. 72, 545 (2000).

[4] S. Adachi, P. Kumbhakar, and T. Kobayashi, Opt. Lett. 29, 1150 (2004).

[5] K. Yamane, Z. Zhang, K. Oka, R. Morita, M. Yamashita, and A. Suguro, Opt. Lett. 28, 2258 (2003).

[6] D. B. Milosevic, G. G. Paulus, D. Baner, and W. Becker, J. Phys. B 39, R203 (2006).

[7] J. J. Carrera, X. M. Tong, and Shih-I Chu, Phys. Rev. A 74, 023404 (2006)

[8] T. Nakajima and S. Watanabe, Phys. Rev. Lett. 96, 213001 (2006).

[9] T. Nakajima and S. Watanabe, Opt. Lett. 31, 1920 (2006).
[10] S. Chelkowski and A. D. Bandrauk, Phys. Rev. A 65, 061802(R) (2002).

[11] Q. Su and J. H. Eberly, Phys. Rev. A 44, 5997 (1991).

[12] X. M. Zhang, J. T. Zhang, L. H. Bai, Q. H. Gong, and Z. Z. Xu, Opt. Express 13, 8708 (2005).

[13] T. Nakajima and G. Buica, Phys. Rev. A 74, 023411 (2006).

[14] X. Tang, H. Rudolph, and P. Lambropoulos, Phys. Rev. Lett. 65, 3269 (1990).

[15] W. Becker, S. Long, and J. K. McIver, Phys. Rev. A 50, 1540 (1994).

[16] W. G. Greenwood and J. H. Eberly, Phys. Rev. A 43, 525 (1991).

[17] A. Cionga, F. Ehlotzky, M. Florescu, and M. Iacomi, J. Phys. B 34, 3951 (2001).

[18] R. Shakeshaft and X. Tang, Phys. Rev. A 36, 3193 (1987). 